\title{
Structural and functional brain asymmetries in the early phases of life: a scoping review
}

\author{
Patrizia Bisiacchi ${ }^{1,2}$ E Elisa Cainelli ${ }^{1}$
}

Received: 26 December 2020 / Accepted: 7 March 2021 / Published online: 18 March 2021

(c) The Author(s) 2021

\begin{abstract}
Asymmetry characterizes the brain in both structure and function. Anatomical asymmetries explain only a fraction of functional variability in lateralization, with structural and functional asymmetries developing at different periods of life and in different ways. In this work, we perform a scoping review of the cerebral asymmetries in the first brain development phases. We included all English-written studies providing direct evidence of hemispheric asymmetries in full-term neonates, foetuses, and premature infants, both at term post-conception and before. The final analysis included 57 studies. The reviewed literature shows large variability in the used techniques and methodological procedures. Most structural studies investigated the temporal lobe, showing a temporal planum more pronounced on the left than on the right (although not all data agree), a morphological asymmetry already present from the 29th week of gestation. Other brain structures have been poorly investigated, and the results are even more discordant. Unlike data on structural asymmetries, functional data agree with each other, identifying a leftward dominance for speech stimuli and an overall dominance of the right hemisphere in all other functional conditions. This generalized dominance of the right hemisphere for all conditions (except linguistic stimuli) is in line with theories stating that the right hemisphere develops earlier and that its development is less subject to external influences because it sustains functions necessary to survive.
\end{abstract}

Keywords Lateralization $\cdot$ Hemispheric specialization $\cdot$ Neonate $\cdot$ Premature $\cdot$ Foetus $\cdot$ Newborn

\section{Introduction}

Asymmetry characterizes the brain, with the right frontal and the left occipital lobes extending across the midline, a phenomenon often called the Yakovlevian torque (LeMay 1976). Asymmetries are present at all levels of structure and function, including regional volumes (Esteves et al. 2019), cortical thickness (Kong et al. 2018), connectivity (Thiebaut de Schotten et al. 2011), cellular and molecular organization (Chance 2014), neurite density (Schmitz et al. 2019) and surface area and gyrification (Chiarello et al. 2016). The most studied asymmetrical function is language, processed in more than $80 \%$ of individuals in the left hemisphere (Esteves et al. 2020). This hemispheric specialization appears to

Patrizia Bisiacchi

patrizia.bisiacchi@unipd.it

1 Department of General Psychology, University of Padova, Via Venezia, 8, 35121 Padova, Italy

2 Padova Neuroscience Centre, PNC, Padova, Italy be functionally relevant, having been demonstrated to be advantageous in the execution of verbal tasks regardless of the direction of asymmetry (Hirnstein et al. 2014). Processing asymmetry has been observed in a broad range of functions, such as episodic memory (Habib et al. 2003), pseudoneglect (Zago et al. 2017), emotional valence (Brunoni et al. 2016), impulsivity (Gordon 2016), risk-taking (Telpaz and Yechiam 2014), and face processing (Zhen et al. 2015).

However, the process of specialization in one cerebral hemisphere (lateralization) is poorly understood. The hemispheric specialization is grounded in intra-hemispheric white matter connections, supported by associative bundles and inter-hemispheric connections between cortical areas located in mirrored positions (homotopic) through the corpus callosum fiber tracts (Ocklenburg et al. 2016). The possibility of associating these structural characteristics with functional correlates has only become possible relatively recently, with the advent of non-invasive neuroimaging methods. However, anatomical asymmetries explain only a fraction of functional variability in lateralization, and this may be associated with 
the fact that structural and functional asymmetries develop at different periods of life and in different ways.

The presence of asymmetries early in development have been investigated by observing foetuses' or neonates' movements, one of the most important manifestations of lateralization. Behavioral studies using ultrasound (US) observations of arm movements suggest the existence of motor lateralization as early as 12-27 weeks of gestation (McCartney and Hepper 1999). Furthermore, evidence for motor lateralization in the neonatal period has been associated with handedness (Cioni and Pellegrinetti 1982) and grasping strength (Tan et al. 1993).

Structural asymmetries were established in the foetal period in the 1970s, when neuropathological studies highlighted some larger areas (Heschl's gyrus and planum temporale) in post-mortem foetuses on the left side (Witelson and Pallie 1973; Chi et al. 1977; Wada 1977).

In recent years, functional image-mapping techniques have emerged as a more sophisticated methodology, allowing researchers to study in vivo brain development; hemispheric asymmetries throughout the life span have been reported, with leftward and rightward asymmetries changing among brain structures at various ages (Matsuzawa et al. 2001; Andescavage et al. 2017). The emergence of asymmetries in the temporal lobes' morphological development has been described as a major sign of lateralization. The most prominent asymmetry involves the peri-Sylvian region and superior temporal sulcus. Interhemispheric differences have been noted in newborn and young infants (Seidenwurm et al. 1985), with dynamic changes through childhood and adulthood (Sowell et al. 2002; Shaw et al. 2009; Fu et al. 2020).

However, most neuroimaging studies have been conducted in children older than age 4 due to the inherent challenge of acquiring data from younger infants. Furthermore, most studies have focused on cortical structures, while deep subcortical grey and white matter have been neglected. Thus, important questions about early brain maturation and hemispheric asymmetries remain unaddressed.

The third trimester of gestation and the neonatal period are the most important developmental periods for the formation of cerebral pathways in terms of path finding, target selection, and growing into the cortical plate (Suppiej et al. 2012). An essential feature of the third trimester of gestation is the transient organization of neuronal circuitry and foetal brain lamination (Kostović and Judaš 2006). This transient organization is supported by the subplate's presence, the most prominent lamina on foetal brain histology, known to disappear at the end of the first year of postnatal life. At this stage of development, the major foetal zones are the cortical plate, subplate, intermediate zone, germinal matrix, deep grey nuclei, and ventricles.
The third trimester comprises the period between 20 and 45 weeks' gestation; based on the major characteristic of the transient pattern of organization, it can be divided into four broadly defined phases: foetal (below 24 postconception weeks [PCW]), early preterm (24-32 PCW), late preterm (33-35 $\mathrm{PCW})$, and neonatal phases (36-45 PCW).

In this work, we aim to provide an overview of the evidence on cerebral asymmetries in the early development stage. We will review all published articles on the neonatal period (1-28 days of life, 36-45 PCW) and the third trimester of gestation (studies on foetuses during pregnancy and infants born preterm in the absence of medical or neurological complications). Premature infants are in vivo models of foetuses in the third trimester of gestation. However, in the absence of medical or neurological complications, "healthy" premature infants do not exist: Prematurity is a risk factor per sé. Therefore, studies on premature infants will be analyzed separately.

\section{Methods}

Scoping reviews are ideal to determine the coverage of a body of literature on a given topic and give a clear indication of the volume of literature and studies available as well as an overview (broad or detailed) of its focus (Munn et al. 2018). Furthermore, scoping reviews can report on the types of evidence in a specific field and how further research may be done on a more specific question. According to the general purpose of scoping reviews, we aimed to identify and map the available evidence on hemispheric asymmetries in the earliest developmental stages.

Our scoping review focused on published works conducted on the first phases of brain development in the third trimester of gestation (studies on foetuses and infants born preterm) and the neonatal period (1-28 days of life or up to $45 \mathrm{PCW}$ ). Studies on at-risk populations or children suffering from pathological conditions were not included; prematurity is an at-risk condition. Still, studies on premature infants were included regardless of whether participants reported additional medical or neurological complications (e.g., intrauterine growth restriction, genetic syndromes, neonatal encephalopathy, intraventricular hemorrhage, periventricular cystic leukomalacia, or the occurrence of seizures).

The scoping review was performed using the PRISMAScR checklist for preferred reporting items for systematic reviews and meta-analysis extension for scoping reviews (Tricco et al. 2018). The resources obtained from this study are available as supplementary material. 


\section{Assessment of methodological quality}

In the current study, we included all papers investigating hemispheric asymmetries in foetuses and premature and full-term neonates, regardless of the technique, methodology of acquisition, pre-processing, or processing algorithms used. Where possible, structural and functional studies were divided into separate sections.

According to the scoping review aims, we did not produce a critically appraised and synthesized result/answer to a particular question; we rather aimed to provide an overview of the evidence in the field. Therefore, we did not perform a structured assessment of methodological limitations or risk of bias of the studies included. However, each work was assessed critically, and the weaknesses and strengths guided us in interpreting the results.

\section{Eligibility criteria}

To be included in the review, papers needed to measure hemispheric asymmetries. Peer-reviewed journal papers were included if participants were foetuses or premature or full-term infants from birth to $45 \mathrm{PCW}$ or 28 days of life; cerebral asymmetries were measured independently of the methodology used; and they were written in English. Behavioral studies on motor lateralization, single cases, animal studies, or studies on at-risk populations or children suffering from pathological conditions were excluded (for the same reason, post-mortem studies were not included). We also excluded works that did not fit into the revision's conceptual framework and those that did not include hemispheric asymmetries in the title, keywords, or abstract. Finally, studies on a wider range of ages than that selected were only included if the results at various ages were clearly stated and differentiated. No limitations were put on the publication year range.

\section{Information sources}

We conducted our search in September 2020, and the search strategies were drafted by two independent neuropsychologists (P.B. and E.C.). We searched PubMed and SCOPUS (Elsevier API) bibliographic databases (which include most of the EMBASE database, https://www.elsevier.com/solut ions/embase-biomedical-research). The search was conducted using the following string: ("asymmetry" OR "laterality") AND ("cerebral" OR "brain" OR "hemispheric") AND ("newborn" OR "neonatal" OR "preterm" OR "fetal" OR "foetal"), which returned a total of 2238 results on
Scopus and 1712 on PubMed. There were no internal duplicates within either database. The final search results were exported for storage and remotion of duplications into the Mendeley bibliographic software package. External duplicates between the databases were removed from the list. The electronic database search was supplemented by screening the reference lists of each retrieved paper and scanning relevant reviews, obtaining an additional 10 works. A total of 2,403 results were screened.

\section{Selection of sources of evidence}

Figure 1 summarizes the following workflow.

The 2403 results were screened based on the article's titles, and 2194 were excluded because they did not focus on cerebral asymmetries, were not original research, or were animal studies. The full texts of the remaining 210 papers were screened, and further exclusion criteria led to the exclusion of 153 additional works. Articles were excluded based on not being original quantitative research $(n=16)$, involving older participants $(n=16)$, being animal studies $(n=13)$, involving participants with pathological conditions or post-mortem studies $(n=24)$, not focusing on hemispheric asymmetries $(n=59)$, being irretrievable $(n=9)$, having only an abstract in English $(n=12)$, or having only the abstract available $(n=4)$. The final analysis included 57 studies.

\section{Data charting process and data items}

A data-charting form was jointly developed by two reviewers (P.B. and E.C.) to determine which variables to extract. The two reviewers independently charted the data, discussed the results, and continuously updated the data charting form in an iterative process. Any disagreements were resolved through discussion between the reviewers. Data from eligible studies were charted using a standardized data abstraction tool designed for this study (see Appendix 1). The tool captured the relevant information on key study characteristics and all techniques used to investigate hemispheric asymmetries.

\section{Data items}

The extraction form is comprised of general and specific characteristics of the articles: reviewer identity, date of reviewing, first author's name, publication year, the title of the article, the journal, the technique used, paradigm and analysis (for functional studies), number of participants, age at recording, participants' status (full-term, 


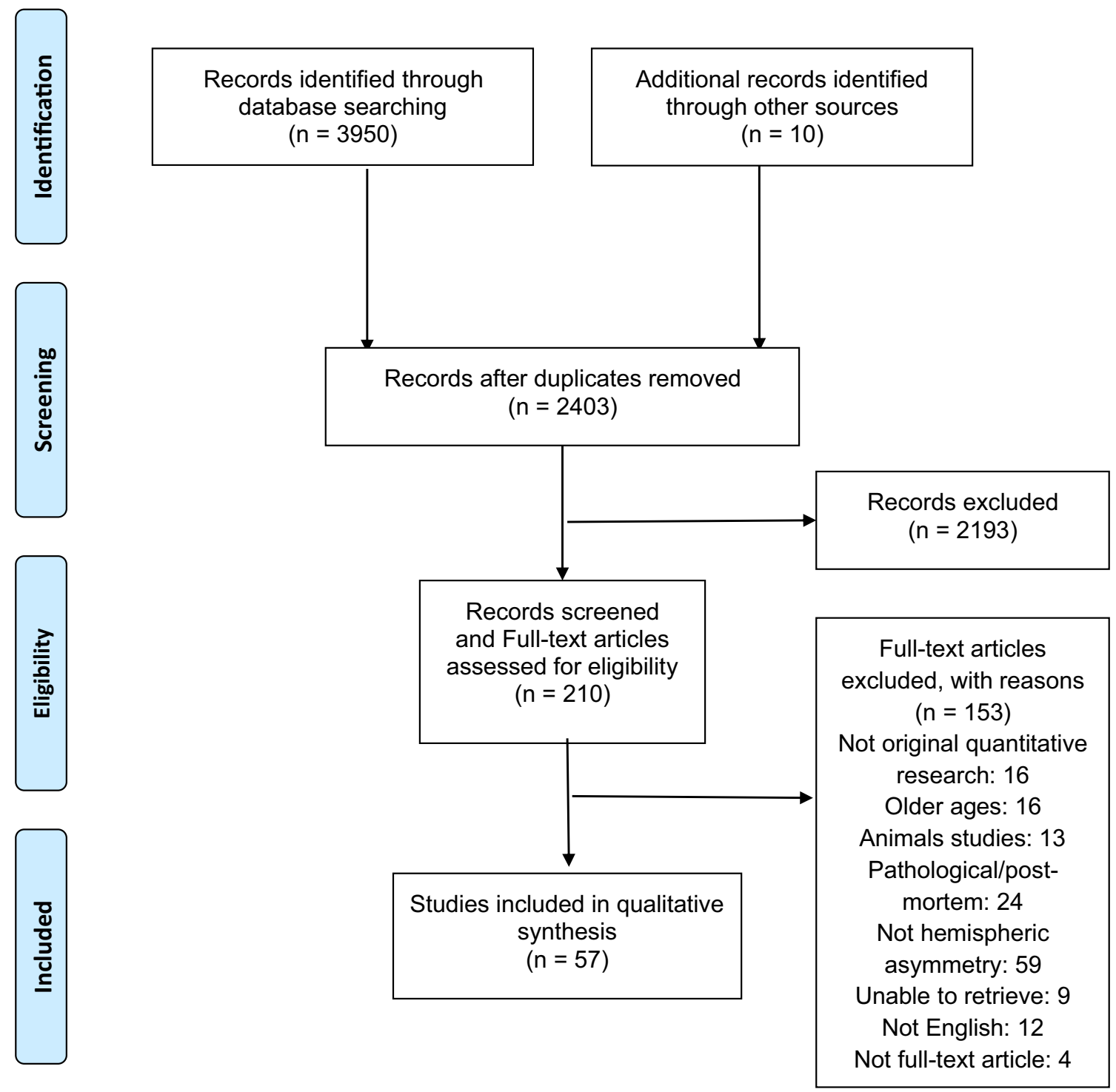

Fig. 1 Workflow of the selection of sources of evidence

premature, or gestation), and a short description of results (see Appendix 1).

\section{Results}

Of the 57 articles eligible for review, 33 were conducted in the neonatal period, 11 during gestation, and 10 before 40 post-conception age (PCA); 3 additional works cover both the neonatal period and the weeks before term PCA.

Figure 2 shows an overview of the number of studies and methodologies used.

Figure 3 shows an overview of the findings on brain asymmetries.

\section{Neonatal period}

Thirty-six studies investigated hemispheric asymmetries in the neonatal period; 8 were mixed studies on preterm infants at term PCA and/or full-term infants, 3 of which covered both the neonatal period and the weeks before term PCA. This section contains the results obtained from fullterm neonates; results obtained from premature infants at term PCA were reported separately in a dedicated paragraph. Articles are reported in Table 1.

The number of studies and methodologies used are shown in Fig. 2 (Panel A and B, "neonates").

The following paragraphs contain the results of studies on structural and functional asymmetries. Results obtained for premature infants will be discussed separately at the end. 


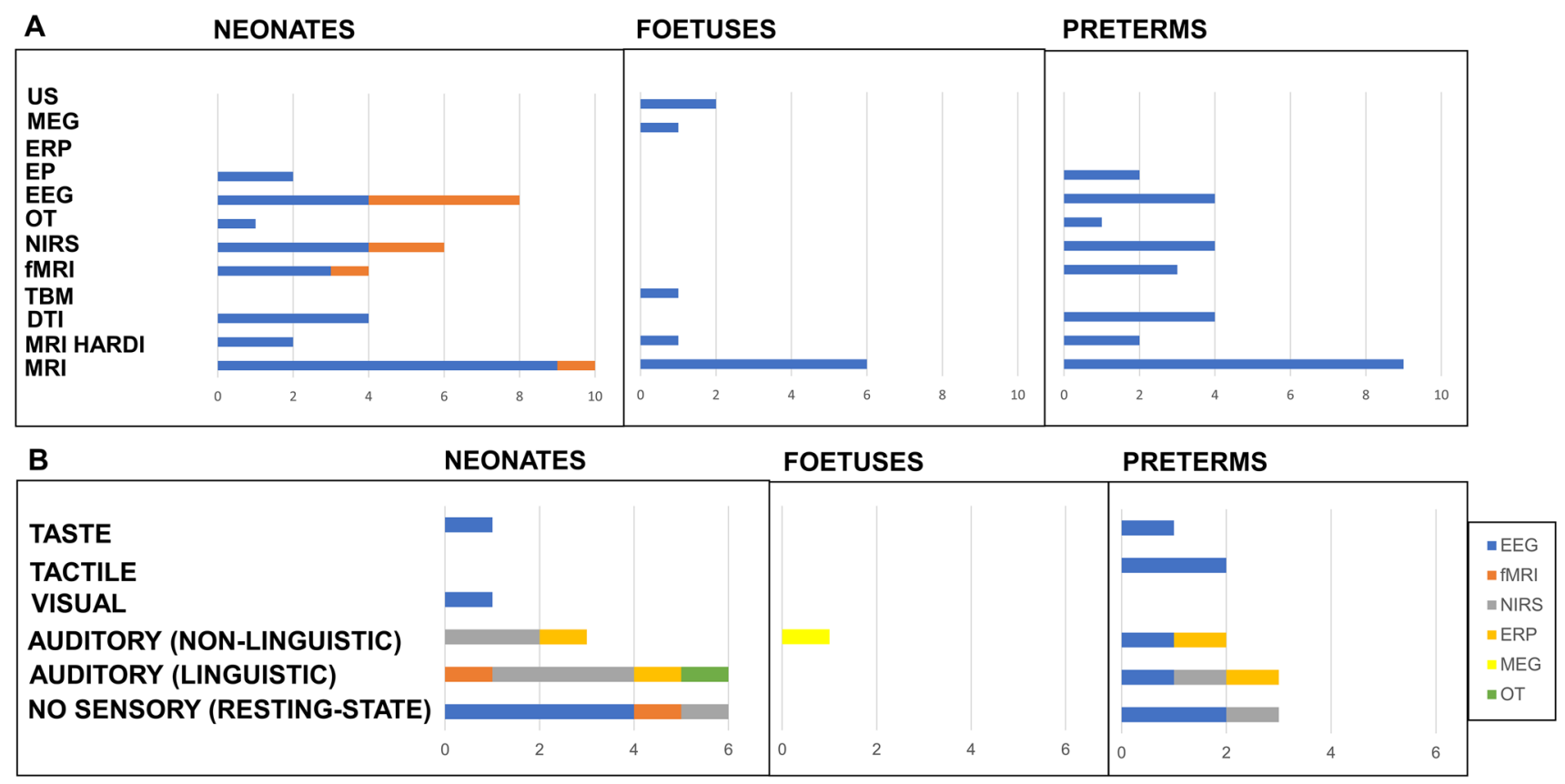

Fig. 2 The number of studies and structural methodologies used in neonates (red represents studies on full-term neonates; blue represents studies on both full-term and preterm neonates at term PCA), foetuses, and preterms (Panel a). The number of studies and functional methodologies used in neonates, foetuses, and preterms (without sensory modality [resting state], auditory [linguistic and nonlinguistic] visual, tactile, and taste stimuli) (Panel b). DTI diffusion

\section{Structural asymmetries in the neonatal period}

Sixteen studies reported structural asymmetries in the brains of full-term neonates. These asymmetries were variable across regions.

Most studies showed a rightward asymmetry of whole subcortical grey matter (Dean et al. 2018) or the hippocampus (Thompson et al. 2009; Ratnarajah et al. 2013) and the putamen (Ratnarajah et al. 2013), but leftward asymmetry has also been reported (Gilmore et al. 2007).

A leftward asymmetry was found for white matter (Gilmore et al. 2007; Dean et al. 2018), but a study focused on arcuate fasciculus found a rightward asymmetry (Wilkinson et al. 2017b); the same author did not find asymmetries on the thalamic-cortical tracts (Wilkinson et al. 2017a).

Data focusing on cortex showed high variability. The temporal lobe is certainly the most investigated, but still with contrasting results: rightward asymmetry was found in the whole temporal lobe (Lehtola et al. 2019), in the superior temporal sulcus (Hill et al. 2010; Li et al. 2014; Lehtola et al. 2019), and the medial temporal and insula (Li et al. 2015). The other authors found a global leftward asymmetry (Gilmore et al. 2007) or one specific to areas such as the planum temporale (Hill et al. 2010; Li et al. 2014), entorhinal cortex, fusiform tensor imaging, EP evoked potential, EEG electroencephalogram, fMRI functional magnetic resonance imaging, HARDI high-angular resolution diffusion imaging, $M E G$ magnetoencephalography, $M R I$ magnetic resonance imaging, NIRS near-infrared spectroscopy, $O T$ optical topography, $P C A$ post-conception age, TBM tensor-based morphometry, US ultrasound

gyrus, insula (Ratnarajah et al. 2013), and the superior temporal sulcus (Duan et al. 2019).

Vannucci and colleagues (2019) found global rightward asymmetry for the frontal lobe, while other researchers indicated a leftward one (Gilmore et al. 2007; Li et al. 2015). Ratnarajah and colleagues found a rightward asymmetry in the cingulate cortex and the gyrus rectus, but a leftward one in the precentral gyrus (Ratnarajah et al. 2013).

A global leftward asymmetry was found for the parietal (Gilmore et al. 2007; Lehtola et al. 2019) and occipital (Gilmore et al. 2007; Lehtola et al. 2019; Vannucci et al. 2019) cortices or specific to the precuneus in the parietal lobe (Ratnarajah et al. 2013). Li and colleagues (2014) found a rightward asymmetry of the parieto-occipital sulcus.

A leftward asymmetry of the ventricles was found in two studies by Gilmore and colleagues (2004, 2007).

Results on structural brain asymmetries are shown in Fig. 3 (above, "neonates"). 


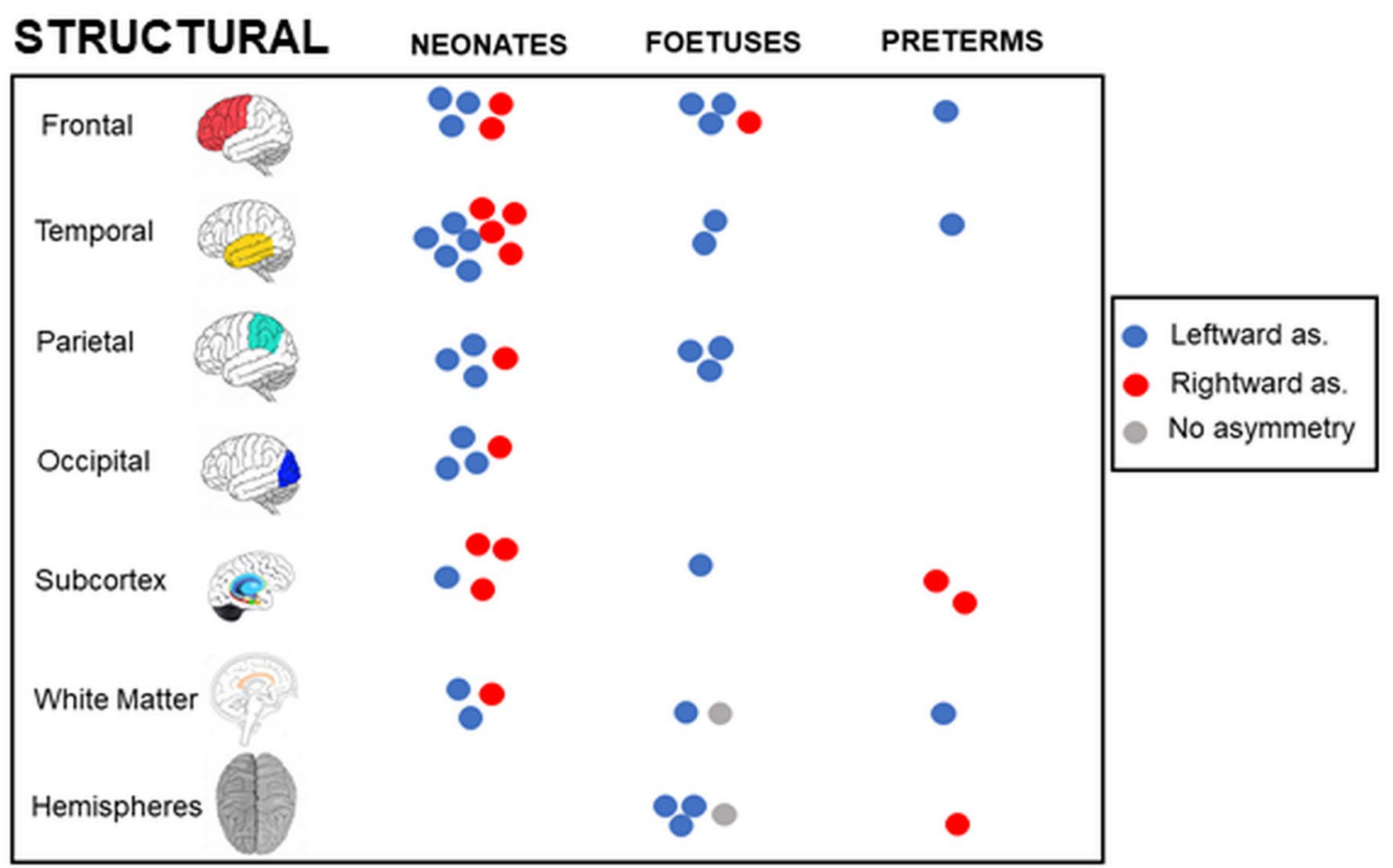

\section{FUNCTIONAL}

Resting state
Auditory
(linguistic)
$\begin{aligned} & \text { Auditory } \\ & \text { (non-linguistic) } \\ & \text { Visual }\end{aligned}$
Taste
Tactile

Fig. 3 Overview of the findings on structural and functional brain asymmetries in neonates, foetuses, and preterms

\section{Functional asymmetries in the neonatal period}

Studies on functional asymmetries can be conducted under stimulation or without sensory stimulation, reflecting the brain's endogenous activity (resting state).

We found six studies investigating endogenous cerebral activity without sensory stimulation and 10 with sensory stimulation at full-term.

\section{Functional studies of endogenous activity}

fMRIs demonstrated a stronger correlation of the medial temporal gyrus with other cerebral areas in the left hemisphere compared to the right (Barttfeld et al. 2018). Spectral analysis of the EEGs revealed significantly higher absolute power in the left central region and the right occipital and temporal areas (Corsi-Cabrera et al. 2020), the right centro-occipital areas (Eiselt et al. 1997), or a general rightward dominance in power 
Table 1 All studies investigating hemispheric asymmetries in the neonatal period in full-term neonates and healthy preterm infants as they reach term

Structural asymmetries in the neonatal period

\begin{tabular}{|c|c|c|c|c|c|}
\hline Authors, year & Technique & Participant number & Participant status & Age at recording & Study design \\
\hline Gilmore et al. (2004) & 3 T MRI DTI & 20 & Full-term & Newborn & $\begin{array}{l}\text { Right/left and sex } \\
\text { within-subjects } \\
\text { comparison }\end{array}$ \\
\hline Gilmore et al. (2007) & 3 T MRI & 74 & Full-term & $42.8 \pm 1.6 \mathrm{PCA}$ & $\begin{array}{l}\text { Right/left within-sub- } \\
\text { jects comparison }\end{array}$ \\
\hline $\begin{array}{l}\text { Thompson et al. } \\
\text { (2009) }\end{array}$ & $1.5 \mathrm{~T}$ MRI & $\begin{array}{l}32 \text { full-term } \\
184 \text { preterm }\end{array}$ & $\begin{array}{l}\text { Full-term }(39 \pm 1.2 \\
\text { GA }) \\
\text { Preterm }(27.6 \pm 1.9 \\
\text { GA })\end{array}$ & At term PCA & $\begin{array}{l}\text { Right/left within-sub- } \\
\text { jects comparison } \\
\text { Preterm/full-term } \\
\text { between-subjects } \\
\text { comparison }\end{array}$ \\
\hline Hill et al. (2010) & 3 T MRI & 12 & Full-term & 39 PCA & $\begin{array}{l}\text { Right/left within-sub- } \\
\text { jects comparison }\end{array}$ \\
\hline Liu et al. (2010) & 1.5 T MRI DTI & $37.5 \pm 1.5 \mathrm{PCA}$ & 27 & $30.0 \pm 2.3 \mathrm{GA}$ & $\begin{array}{l}\text { Right/left and age } \\
\text { within-subjects } \\
\text { comparison }\end{array}$ \\
\hline $\begin{array}{l}\text { Ratnarajah et al. } \\
\text { (2013) }\end{array}$ & $1.5 \mathrm{~T} \mathrm{DTI}$ & 124 & Full-term & 5-17 days & $\begin{array}{l}\text { Right/left within-sub- } \\
\text { jects comparison }\end{array}$ \\
\hline Li et al. (2014) & 3 T MRI & 73 & Full-term & $\begin{array}{l}27.3 \pm 13.1 \text { days } \\
1,2 \text { years }\end{array}$ & $\begin{array}{l}\text { Right/left and age } \\
\text { within-subjects } \\
\text { comparison }\end{array}$ \\
\hline Meng et al. (2014) & 3 T MRI & 73 & Full-term & $\begin{array}{l}27.3 \pm 13.1 \text { days } \\
1,2 \text { years }\end{array}$ & $\begin{array}{l}\text { Right/left and age } \\
\text { within-subjects } \\
\text { comparison }\end{array}$ \\
\hline Li et al. (2015) & 3 T MRI & 73 & Full-term & $\begin{array}{l}27.3 \pm 13.1 \text { days } \\
1,2 \text { years }\end{array}$ & $\begin{array}{l}\text { Right/left and age } \\
\text { within-subjects } \\
\text { comparison }\end{array}$ \\
\hline $\begin{array}{l}\text { Wilkinson et al., } \\
(2017 \mathrm{a}, \mathrm{b})\end{array}$ & $\begin{array}{l}3 \text { T MRI HARDI of } \\
\text { arcuate fasciculus }\end{array}$ & 83 & Full-term & 40 PCA-28 years & $\begin{array}{l}\text { Right/left within- } \\
\text { subjects comparison } \\
\text { Age between-sub- } \\
\text { jects comparison }\end{array}$ \\
\hline $\begin{array}{l}\text { Wilkinson et al. } \\
\text { 2017a, b }\end{array}$ & $\begin{array}{l}3 \text { T MRI HARDI of } \\
\text { thalamus }\end{array}$ & $\begin{array}{l}83 \text { in vivo } \\
11 \text { post-mortem } \\
\text { foetuses }\end{array}$ & $\begin{array}{l}\text { Full-term } \\
\text { foetuses }\end{array}$ & $\begin{array}{l}40 \text { PCA-28 years } \\
\text { Post-mortem foetuses }\end{array}$ & $\begin{array}{l}\text { Right/left within- } \\
\text { subjects comparison } \\
\text { Age between-sub- } \\
\text { jects comparison }\end{array}$ \\
\hline Dean et al. (2018) & 3 T MRI & 143 & Full-term & 1 month & $\begin{array}{l}\text { Right/left within-sub- } \\
\text { jects comparison }\end{array}$ \\
\hline Lehtola et al. (2018) & 3 T MRI & 68 & Full-term & $2-5$ PCA & $\begin{array}{l}\text { Right/left within- } \\
\text { subjects comparison } \\
\text { Age between-sub- } \\
\text { jects comparison }\end{array}$ \\
\hline Duan et al. (2019) & 3 T MRI & 595 & Full-term & 36.7-46.5 PCA & $\begin{array}{l}\text { Right/left within-sub- } \\
\text { jects comparison }\end{array}$ \\
\hline Vannucci et al. (2019) & 1.5 and $3 \mathrm{~T}$ MRI & 121 & Full-term & $0-18$ years & $\begin{array}{l}\text { Right/left within-sub- } \\
\text { jects comparison } \\
\text { Age and sex between- } \\
\text { subjects comparison }\end{array}$ \\
\hline \multicolumn{6}{|c|}{ Functional asymmetries in the neonatal period } \\
\hline Croweell et al. (1973) & $\begin{array}{l}\text { EEG repetitive visual } \\
\text { stimulation } \\
\text { Spectral analysis }\end{array}$ & 97 & Full-term & - & $\begin{array}{l}\text { Right/left within-sub- } \\
\text { jects comparison }\end{array}$ \\
\hline $\begin{array}{l}\text { Molfese and Nunez } \\
\text { (1976) }\end{array}$ & AEP & 14 & Full-term & Within $48 \mathrm{~h}$ of birth & $\begin{array}{l}\text { Right/left within-sub- } \\
\text { jects comparison }\end{array}$ \\
\hline $\begin{array}{l}\text { Fox and Davidson } \\
\text { (1986) }\end{array}$ & $\begin{array}{l}\text { EEG } \\
\text { Taste stimuli } \\
\text { Spectral analysis }\end{array}$ & 16 & Full-term & $2-3$ days & $\begin{array}{l}\text { Right/left within-sub- } \\
\text { jects comparison }\end{array}$ \\
\hline
\end{tabular}


Table 1 (continued)

Structural asymmetries in the neonatal period

\begin{tabular}{|c|c|c|c|c|c|}
\hline Authors, year & Technique & Participant number & Participant status & Age at recording & Study design \\
\hline $\begin{array}{l}\text { Majnemer and Rosen- } \\
\text { blatt (1992) }\end{array}$ & SEP & 9 & Full-term & $\begin{array}{l}2-3 \text { days } \\
2 \text { months } \\
6 \text { months }\end{array}$ & $\begin{array}{l}\text { Right/left and age } \\
\text { within-subjects } \\
\text { comparison }\end{array}$ \\
\hline Eiselt et al. (1997) & $\begin{array}{l}\text { EEG } \\
\text { Resting state } \\
\text { Spectral analysis }\end{array}$ & 12 & $\begin{array}{l}6 \text { full-term } \\
7 \text { preterm } \\
(<32 \mathrm{GA})\end{array}$ & $3-8$ days & $\begin{array}{l}\text { Right/left within-sub- } \\
\text { jects comparison } \\
\text { Preterm/full-term } \\
\text { between-subjects } \\
\text { comparison }\end{array}$ \\
\hline Scher et al. (1997) & $\begin{array}{l}\text { EEG } \\
\text { Resting state } \\
\text { Spectral analysis }\end{array}$ & $\begin{array}{l}55 \text { preterm } \\
45 \text { full-term }\end{array}$ & $\begin{array}{l}\text { Preterm } \\
(\leq 32 \mathrm{GA}) \\
\text { Full-term }\end{array}$ & At term PCA & $\begin{array}{l}\text { Right/left within-sub- } \\
\text { jects comparison } \\
\text { Preterm/full-term } \\
\text { between-subjects } \\
\text { comparison }\end{array}$ \\
\hline Pena et al. (2003) & $\begin{array}{l}\text { Optical topography } \\
\text { Infant-directed speech, } \\
\text { reversed, and silence }\end{array}$ & 14 & Full-term & $2-5$ days & $\begin{array}{l}\text { Right/left within-sub- } \\
\text { jects comparison }\end{array}$ \\
\hline Ehberrich et al. (2006) & $\begin{array}{l}1.5 \mathrm{~T} \text { fMRI } \\
\text { Cutaneous and } \\
\text { proprioceptive } \\
\text { stimulation }\end{array}$ & $\begin{array}{l}42 \text { newborn } \\
6 \text { infants }\end{array}$ & $\begin{array}{l}\text { Newborn } \\
\text { infants (3-9 months) }\end{array}$ & $\begin{array}{l}\text { Mean } 42(38-49) \\
\text { PCA }\end{array}$ & $\begin{array}{l}\text { Right/left within-sub- } \\
\text { jects comparison } \\
\text { Newborn/infants } \\
\text { between-subjects } \\
\text { comparison }\end{array}$ \\
\hline $\begin{array}{l}\text { Telkemeyer et al. } \\
\text { (2009) }\end{array}$ & $\begin{array}{l}\text { EEG } \\
\text { NIRS } \\
\text { Structured non-speech } \\
\text { stimuli }\end{array}$ & 34 & Full-term & $2-6$ days & $\begin{array}{l}\text { Right/left within-sub- } \\
\text { jects comparison }\end{array}$ \\
\hline Kotilahti et al. (2010) & $\begin{array}{l}\text { NIRS } \\
\text { Infant-directed speech } \\
\text { and music }\end{array}$ & 13 & Full-term & 37.3-42.3 PCA & $\begin{array}{l}\text { Right/left and condi- } \\
\text { tion within-subjects } \\
\text { comparison }\end{array}$ \\
\hline Gonzales et al. (2011) & $\begin{array}{l}\text { EEG } \\
\text { Resting state } \\
\text { Connectivity }\end{array}$ & 21 & $\begin{array}{l}7: 33-34 \\
7: 38-39 \\
7: 38-39 \\
\text { GA }\end{array}$ & $\begin{array}{l}39-40 \text { PCA } 39-40 \\
\text { PCA } \\
44-45 \text { PCA }\end{array}$ & $\begin{array}{l}\text { Right/left within-sub- } \\
\text { jects comparison } \\
\text { Age between-subjects } \\
\text { comparison }\end{array}$ \\
\hline $\begin{array}{l}\text { Minagawa-Kawai } \\
\text { et al. (2011) }\end{array}$ & $\begin{array}{l}\text { NIRS } \\
\text { Auditory stimuli (vari- } \\
\text { ous durations) }\end{array}$ & 29 & Full-term & $0-5$ days & $\begin{array}{l}\text { Right/left and condi- } \\
\text { tion within-subjects } \\
\text { comparison }\end{array}$ \\
\hline Perani et al. (2011) & $\begin{array}{l}\text { fMRI } \\
\text { Female voice } \\
\text { Connectivity } \\
\text { DTI }\end{array}$ & $\begin{array}{l}15 \text { full-term } \\
\text { adults }\end{array}$ & $\begin{array}{l}\text { Full-term } \\
\text { Adult }\end{array}$ & 2 days & $\begin{array}{l}\text { Right/left within-sub- } \\
\text { jects comparison } \\
\text { Age between-subjects } \\
\text { comparison }\end{array}$ \\
\hline Lin et al. (2013) & $\begin{array}{l}\text { FDNIRS } \\
\text { DCS } \\
\text { Resting state }\end{array}$ & 70 & $\begin{array}{l}55 \text { preterm }(24-42 \\
\text { GA) } \\
15 \text { full-term }\end{array}$ & 3.6 weeks & $\begin{array}{l}\text { Right/left within-sub- } \\
\text { jects comparison } \\
\text { Preterm/full-term and } \\
\text { sex between-subjects } \\
\text { comparison }\end{array}$ \\
\hline Myers et al. (2012) & $\begin{array}{l}\text { EEG } \\
\text { Resting state } \\
\text { Spectral power } \\
\text { Burst detection }\end{array}$ & 252 & $\begin{array}{l}171 \text { preterm } \\
81 \text { full-term }\end{array}$ & 35-52 PCA & $\begin{array}{l}\text { Right/left and preterm/ } \\
\text { full-term between- } \\
\text { subjects comparison }\end{array}$ \\
\hline Bouchon et al. (2015) & $\begin{array}{l}\text { NIRS } \\
\text { Artificial and random } \\
\text { grammar }\end{array}$ & 24 & Full-term & 1-3 days & $\begin{array}{l}\text { Right/left within-sub- } \\
\text { jects comparison }\end{array}$ \\
\hline Kwon et al. (2015) & $\begin{array}{l}3 \mathrm{~T} \text { fMRI } \\
\text { Resting state } \\
\text { Connectivity }\end{array}$ & $\begin{array}{l}26 \text { preterm } \\
25 \text { full-term }\end{array}$ & $\begin{array}{l}\text { Preterm }(500-1500 \mathrm{~g}) \\
\text { Full-term }\end{array}$ & At term PCA & $\begin{array}{l}\text { Right/left within-sub- } \\
\text { jects comparison } \\
\text { Preterm/full-term } \\
\text { between-subjects } \\
\text { comparison }\end{array}$ \\
\hline
\end{tabular}


Table 1 (continued)

\begin{tabular}{|c|c|c|c|c|c|}
\hline \multicolumn{6}{|c|}{ Structural asymmetries in the neonatal period } \\
\hline Authors, year & Technique & Participant number & Participant status & Age at recording & Study design \\
\hline Arimitsu et al. (2018) & $\begin{array}{l}\text { NIRS } \\
\text { Phonetic changes }\end{array}$ & 80 & $\begin{array}{l}60 \text { preterm } \\
(26-41 \mathrm{GA}) \\
20 \text { term }\end{array}$ & 33-41 PCA & $\begin{array}{l}\text { Right/left and } \\
\text { Age between-subjects } \\
\text { comparison }\end{array}$ \\
\hline Barttfeld et al. (2018) & $\begin{array}{l}1.5 \mathrm{~T} \text { fMRI } \\
\text { Resting state }\end{array}$ & 24 & Full-term & $41.1 \pm 1.08$ PCA & $\begin{array}{l}\text { Right/left within-sub- } \\
\text { jects comparison }\end{array}$ \\
\hline $\begin{array}{l}\text { Corsi-Cabrera et al. } \\
\text { (2019) }\end{array}$ & $\begin{array}{l}\text { EEG } \\
\text { Resting state } \\
\text { Spectral analysis }\end{array}$ & 60 & Full-term & $\begin{array}{l}41,42,43,44,45 \\
\text { PCA }\end{array}$ & $\begin{array}{l}\text { Right/left within-sub- } \\
\text { jects comparison } \\
\text { Age between-subjects } \\
\text { comparison }\end{array}$ \\
\hline
\end{tabular}

$A E P$ auditory-evoked potentials, $D C S$ diffuse correlation spectroscopy, $E E G$ electroencephalogram, FDNIRS frequency domain near-infrared spectroscopy, GA gestational age, HARDI high-angular resolution diffusion, MRI magnetic resonance imaging, $P C A$ post-conception age, SEP somatosensory-evoked potentials

below $13 \mathrm{~Hz}$ between 35 and 45 PCW (Myers et al. 2012). The behavioral state seems to affect spectral content in the right and left hemispheres: During active sleep, spectral power is higher in right posterior regions. During quiet sleep, spectral power is higher in the frontal areas of both hemispheres than the posterior (Scher et al. 1997). A rightward dominance in the temporal and parietal metabolism has been found using NIRS and diffuse correlation spectroscopy (Lin et al. 2013).

\section{Functional studies under stimulation}

Speech stimuli (grammar and infant-directed speech) evoked leftward responses using NIRS (Kotilahti et al. 2010; Bouchon et al. 2015), optical topography (Peña et al. 2003), and auditory-evoked potentials (Molfese et al. 1976). By contrast, using fMRI and female voices, Perani and colleagues found a rightward asymmetry (2011). In addition, non-linguistic aspects of speech stimuli, such as prosody, evoked a rightward dominance based on fNIRS (Arimitsu et al. 2018).

Non-speech auditory stimulation evoked a rightward asymmetry using auditory-evoked potentials and NIRS (Telkemeyer et al. 2009) as well as auditory-evoked potentials alone (Molfese et al. 1976). By contrast, using NIRS, MinagawaKawai and colleagues did not find asymmetries (2011).

Finally, spectral EEG in response to rhythmic visual stimuli (Crowell et al. 1973) and taste (Fox and Davidson 1986) showed rightward asymmetry.

Results on functional brain asymmetries are shown in Fig. 3 (below, "neonates").

\section{Gestational period}

We found 11 studies exploring hemispheric asymmetries in foetuses during pregnancy (Table 2). All but one were structural investigations of hemispheric asymmetries. Articles are reported in Table 2.

The number of studies and methodologies used are shown in Fig. 2 (Panel A and B, "foetuses").

\section{Structural asymmetries in the gestational period}

Data showed that during primary gyrogenesis, the right hemisphere undergoes cortical folding earlier than the left (Rajagopalan et al. 2011, 2012; Kasprian et al. 2011; Habas et al. 2012), with the appearance of the right superior temporal sulcus by 23 (Kasprian et al. 2011) or 24 gestational weeks (Habas et al. 2012). However, some specific areas of the left parahippocampal cerebral mantle have been reported to be larger on the left than the right (Rajagopalan et al. 2011), and a certain asymmetry pattern has been established with a longer left temporal lobe (Kasprian et al. 2011).

However, there is also contrasting evidence. A small but significant number of larger left hemispheres have been noted, both in cortical grey matter and deep subcortical 
Table 2 Studies investigating hemispheric asymmetries in foetuses during pregnancy

\begin{tabular}{|c|c|c|c|c|}
\hline \multicolumn{5}{|c|}{ Structural asymmetries in the gestational period } \\
\hline Authors, year & Technique & Age at recording & Participant number & Study design \\
\hline Hering-Hanit et al. (2001) & US & $20-22$ GA & 102 & Right/left within-subjects comparison \\
\hline Kivilevitch et al. (2010) & US & 19-28 GA & 406 & Right/left within-subjects comparison \\
\hline Kasprian et al. (2011) & $1.5 \mathrm{~T}$ MRI & $18-37$ GA & 197 & $\begin{array}{l}\text { Right/left within-subjects comparison } \\
\text { Age between-subjects comparison }\end{array}$ \\
\hline Rajagopalan et al. (2011) & $1.5 \mathrm{~T}$ MRI & $20-28 \mathrm{GA}$ & 38 & $\begin{array}{l}\text { Right/left within-subjects comparison } \\
\text { Age between-subjects comparison }\end{array}$ \\
\hline Scott et al. (2011) & $1.5 \mathrm{~T}$ MRI & $20.57-31.14 \mathrm{GA}$ & 39 & $\begin{array}{l}\text { Right/left within-subjects comparison } \\
\text { Age between-subjects comparison }\end{array}$ \\
\hline Habas et al. (2012) & $1.5 \mathrm{~T}$ MRI & $20-28 \mathrm{GA}$ & 38 & $\begin{array}{l}\text { Right/left within-subjects comparison } \\
\text { Age between-subjects comparison }\end{array}$ \\
\hline Rajagopalan et al. (2012) & Tensor-based morphometry & $20.57-27.86$ GA & 38 (40 scans) & $\begin{array}{l}\text { Right/left within-subjects comparison } \\
\text { Age between-subjects comparison }\end{array}$ \\
\hline Song et al. (2015) & 3 T MRI HARDI & 15 GA-3 years & $\begin{array}{l}23 \text { (11 post-mortems, } \\
12 \text { foetuses) }\end{array}$ & $\begin{array}{l}\text { Right/left within-subjects comparison } \\
\text { Age between-subjects comparison }\end{array}$ \\
\hline Andescavage et al. (2017) & $1.5 \mathrm{~T}$ MRI & 18-39 GA & 166 & $\begin{array}{l}\text { Right/left within-subjects comparison } \\
\text { Age between-subjects comparison }\end{array}$ \\
\hline Vasung et al. (2019) & 3 T MRI & $16.43-36.86 \mathrm{GA}$ & 42 & Right/left within-subjects comparison \\
\hline \multicolumn{5}{|c|}{ Functional asymmetries in the gestational period } \\
\hline Schleussner et al. (2004) & $\begin{array}{l}\text { MEG } \\
500 \mathrm{~Hz}\end{array}$ & 3rd trimester & 38 (53 scans) & Right/left within-subjects comparison \\
\hline
\end{tabular}

GA gestational age, HARDI high-angular resolution diffusion, $M E G$ magnetoencephalography, $M R I$ magnetic resonance imaging, US ultrasound sonography

structures (Andescavage et al. 2017). The larger volume of the left hemisphere was also confirmed by ultrasound examinations (Hering-Hanit et al. 2001; Kivilevitch et al. 2010).

Other cerebral areas, such as some regions of the frontal and parietal lobes (the inferior frontal gyrus and frontal operculum) showed leftward dominance (Rajagopalan et al. 2011, 2012; Vasung et al. 2020), while the orbitofrontal cortex showed a rightward one (Vasung et al. 2020).

A leftward hemispheric asymmetry was found in the inferior longitudinal fasciculus of the white matter. In contrast, asymmetry of pathways associated with higher-order cognitive functions, such as the arcuate fasciculus, was not observed (Song et al. 2015).

No significant differences in brain hemispheric symmetries were found by Scott and colleagues (2011).

Results on structural brain asymmetries are shown in Fig. 3 (above, "foetuses").

\section{Functional asymmetries in the gestational period}

In a unique study, researchers investigated the functional asymmetries in foetuses by recording magnetoencephalography during auditory stimulation (Schleussner et al. 2004). Researchers found a delay in the latency of the cortical auditory responses in the left hemisphere, suggesting earlier maturation of right brain areas.

Results on functional brain asymmetries are shown in Fig. 3 (below, "foetuses").

\section{Premature infants before term PCA}

We found 13 studies on hemispheric asymmetries in premature infants before term PCA. These studies showed great variability in methodologies and techniques.

In contrast to studies conducted in the neonatal period or during pregnancy, most studies on premature infants before term PCA were based on functional asymmetries. Articles are reported in Table 3.

The number of studies and methodologies used are shown in Fig. 2 (Panel A and B, "preterms").

\section{Structural asymmetries in premature infants before term PCA}

We found three studies investigating structural asymmetries in premature infants. A larger right hemisphere has been reported, as well as the fact that gyral complexity emerges earlier on the right, particularly in the superior temporal sulcus (Dubois et al. 2008, 2010). The same author also 
Table 3 Studies investigating hemispheric asymmetries in premature infants before term PCA

Structural asymmetries in premature infants before term PCA

\begin{tabular}{|c|c|c|c|c|c|}
\hline Authors, year & Technique & Age at recording & Participant number & Gestational age & Study design \\
\hline Dubois et al. (2008) & $1.5 \mathrm{~T}$ MRI & $31.1 \pm 2.4$ PCA & 35 & $30.0 \pm 2.5 \mathrm{GA}$ & $\begin{array}{l}\text { Right/left within-sub- } \\
\text { jects comparison } \\
\text { Age and sex between- } \\
\text { subjects comparison }\end{array}$ \\
\hline Dubois et al. (2010) & 1.5 T MRI & $31.5 \pm 2.4$ PCA & 25 & $26-36 \mathrm{GA}$ & $\begin{array}{l}\text { Right/left within-sub- } \\
\text { jects comparison } \\
\text { Age between-subjects } \\
\text { comparison }\end{array}$ \\
\hline Guo et al. (2015) & $\begin{array}{l}1.5 \mathrm{~T} \text { MRI on the hip- } \\
\text { pocampus }\end{array}$ & $\begin{array}{l}\text { Birth } \\
\text { at term PCA }\end{array}$ & 197 & $24-32 \mathrm{GA}$ & $\begin{array}{l}\text { Right/left and age } \\
\text { within-subjects com- } \\
\text { parison }\end{array}$ \\
\hline \multicolumn{6}{|c|}{ Functional asymmetries in premature infants before term PCA } \\
\hline Eiselt et al. (1997) & $\begin{array}{l}\text { EEG } \\
\text { Resting state } \\
\text { Spectral analysis }\end{array}$ & 12 & $\begin{array}{l}6 \text { full-term } \\
7 \text { preterm } \\
(<32 \mathrm{GA})\end{array}$ & 3-8 days & $\begin{array}{l}\text { Right/left within-sub- } \\
\text { jects comparison } \\
\text { Preterm/full-term } \\
\text { between-subjects } \\
\text { comparison }\end{array}$ \\
\hline Mento et al. (2010) & $\begin{array}{l}\text { ERP } \\
\text { Auditory oddball }\end{array}$ & 35 PCA & 34 & 24-34 GA & Cluster analysis \\
\hline Lin et al. (2013) & $\begin{array}{l}\text { FDNIRS } \\
\text { DCS } \\
\text { Resting state }\end{array}$ & 70 & $\begin{array}{l}55 \text { preterm }(24-42 \\
\text { GA) } \\
15 \text { full-term }\end{array}$ & 3.6 weeks & $\begin{array}{l}\text { Right/left within-sub- } \\
\text { jects comparison } \\
\text { Preterm/full-term and } \\
\text { sex between-subjects } \\
\text { comparison }\end{array}$ \\
\hline Barlow et al. (2014) & $\begin{array}{l}\text { aEEG orocutaneous st. } \\
\text { Non-stimulation } \\
\text { Spectral analysis }\end{array}$ & 32 PCA & 22 & $28.56 \mathrm{GA}$ & $\begin{array}{l}\text { Right/left within-sub- } \\
\text { jects comparison } \\
\text { Conditions between- } \\
\text { subjects comparison }\end{array}$ \\
\hline Maitre et al. (2014) & $\begin{array}{l}\text { ERP } \\
\text { Speech } \\
\text { Psychomotor assess- } \\
\text { ment }\end{array}$ & $>32$ PCA & 57 & 24-40 GA & $\begin{array}{l}\text { Right/left and outcome } \\
\text { within-subjects com- } \\
\text { parison } \\
\text { GA/PCA between-sub- } \\
\text { jects comparison }\end{array}$ \\
\hline Song et al. (2014) & $\begin{array}{l}\text { EEG } \\
\text { Pulsed orocutaneous } \\
\text { stimulation } \\
\text { Spectral edge fre- } \\
\text { quency (SEF) }\end{array}$ & $32.2 \pm 1.09$ PCA & 22 & $\begin{array}{l}28.6 \pm 2.1 \mathrm{GA} \\
1,230 \pm 338 \mathrm{~g}\end{array}$ & $\begin{array}{l}\text { Right/left within-sub- } \\
\text { jects comparison } \\
\text { Condition between- } \\
\text { subjects comparison }\end{array}$ \\
\hline Kaminska et al. (2017) & $\begin{array}{l}\text { EEG-MRI } \\
\text { Auditory stimuli } \\
\quad \text { (click) } \\
\text { Evoked potentials }\end{array}$ & 30-38 PCA & 30 & $26-36 \mathrm{GA}$ & $\begin{array}{l}\text { Right/left within-sub- } \\
\text { jects comparison } \\
\text { Age between-subjects } \\
\text { comparison }\end{array}$ \\
\hline Arimitsu et al. (2018) & $\begin{array}{l}\text { NIRS } \\
\text { Phonetic changes of } \\
\text { speech }\end{array}$ & 33-41 PCA & 80 & $\begin{array}{l}60 \text { preterm } \\
(26-41 \mathrm{GA}) \\
20 \text { full-term }\end{array}$ & $\begin{array}{l}\text { Right/left within-sub- } \\
\text { jects comparison } \\
\text { Age between-subjects } \\
\text { comparison }\end{array}$ \\
\hline Cainelli et al. 2019 & $\begin{array}{l}\text { EEG } \\
\text { Resting state } \\
\text { Connectivity }\end{array}$ & 35 PCA & 16 & $\begin{array}{l}\text { 8: 23-28 GA } \\
8: 34-35 \text { GA }\end{array}$ & $\begin{array}{l}\text { Right/left within-sub- } \\
\text { jects comparison } \\
\text { GA between-subjects } \\
\text { comparison }\end{array}$ \\
\hline $\begin{array}{l}\text { Daneshvarfard et al. } \\
\text { (2019) }\end{array}$ & $\begin{array}{l}\text { EEG } \\
\text { Repetitive syllables } \\
\text { Spectral analysis }\end{array}$ & 29.57-34.14 PCA & 16 & $29.57-34.14 \mathrm{GA}$ & $\begin{array}{l}\text { Right/left within-sub- } \\
\text { jects comparison } \\
\text { Age between-subjects } \\
\text { comparison }\end{array}$ \\
\hline
\end{tabular}

aEEG amplitude-integrated electroencephalogram, DCS diffuse correlation spectroscopy, ERP event-related potentials, FDNIRS frequency domain near-infrared spectroscopy, GA gestational age, $M R I$ magnetic resonance imaging, $P C A$ post-conception age 
reported larger regions posterior and anterior to the Sylvian fissure (respectively close to planum temporale and Broca's region) on the left side (Dubois et al. 2010). The right hippocampus showed a rightward asymmetry (Guo et al. 2015).

Results on structural brain asymmetries are shown in Fig. 3 (above, "preterms").

\section{Functional asymmetries in premature infants before term PCA}

We found three studies investigating endogenous cerebral activity without sensory stimulation and seven under sensory stimulation (auditory [speech and non-speech] and tactile) in premature infants. Some studies have been conducted to investigate the presence of functional asymmetries in the resting state and under stimulation.

Studies investigating cerebral activity at rest converged to identify a rightward dominance, supported by the use of various techniques (spectral and connectivity analysis of the EEG, NIRS, and DCS) (Eiselt et al. 1997; Lin et al. 2013; Cainelli et al. 2020b). Results were mainly found in centralposterior and temporal areas, but not in frontal areas, according to the slower development rate of the frontal areas (Lin et al. 2013).

In addition, the response to stimulation appeared to evoke a greater response in the right hemisphere for non-speech stimuli (such as oddball paradigms and auditory clicks) (Mento et al. 2010; Kaminska et al. 2018) or non-linguistic aspects of speech stimuli, such as prosody, but only preterm at later PCAs (Arimitsu et al. 2018). Phonemic contrast (Arimitsu et al. 2018) and consonant-vowel syllable (Maitre et al. 2014) stimulation evoked leftward dominance. Furthermore, the asymmetry correlated with communicative abilities at 6 and 12 months (Maitre et al. 2014). However, a rightward asymmetry has also been found to detect repetitive syllabic stimuli (Daneshvarfard et al. 2019). Orocutaneous stimulation gave divergent results in terms of left (Barlow et al. 2014) or right (Song et al. 2014) dominance.

Results on functional brain asymmetries are shown in Fig. 3 (below, "preterms").

\section{The case of premature infants at term-corrected age}

Preterm and full-term infants demonstrated rightward hippocampal asymmetry (Thompson et al. 2009; Guo et al. 2015), but preterm infants tended to have less asymmetrical hippocampi than full-term infants (Thompson et al. 2009). A white-matter tract investigation using DTI in premature infants at term showed a leftward asymmetry in the parietotemporal part of the superior longitudinal fasciculus and a trend toward leftward asymmetry in diffusion indices in the corticospinal tract. Furthermore, a leftward volume asymmetry has been found in the motor part of the superior thalamic radiations ( $\mathrm{Li}$ et al. 2015).

From a functional point of view, it has been reported that premature infants at term-corrected age show lower spectra compared to full-term neonates in specific regions, particularly in the left parasagittal and the sagittal regions (Scher et al. 1997). Furthermore, they showed lower coherence in the left frontopolar-centrotemporal and right occipital-centrotemporal regions in the beta band during active sleep (González et al. 2011). Using fMRI connectivity measures at rest in very preterm subjects (birth weight $500-1500 \mathrm{~g}$ ) at term equivalent age, premature neonates revealed more significant differences in cerebral lateralization in the left hemisphere language regions than controls (Kwon et al. 2015).

Results on brain asymmetries are shown in Fig. 3 (above, "preterms").

\section{Sex differences}

Of the 57 works, 22 explored the presence of sexual dimorphism in hemispheric asymmetries explicitly (10 on fullterm infants, 6 on foetuses, and 6 on premature infants). Only five found sex-based differences. The differences detected are difficult to compare because studies focused on different targets, but the results appear to be quite contrasting. For example, it has found a more predominant leftward lateralization during gestation for males (Kivilevitch et al. 2010), rightward in premature infants for females (Dubois et al. 2008), and males (Lin et al. 2012).

\section{Discussion}

We performed a scoping review of the existing literature on hemispheric asymmetries in the first brain development phases. We reviewed studies using neuroimaging methods, which provide direct evidence on hemispheric, structural, and/or functional asymmetries, in full-term neonates, foetuses during pregnancy, and premature infants, both at term PCA and before. Given the low number of studies, we did not select a specific year range. Rather, we collected all the available evidence, yielding 57 studies.

The reviewed literature shows high variability in techniques and methodological procedures. Most studies based on the neonatal period and gestation were structural investigations, while most of those conducted in premature infants were functional. Finally, we searched for sexual dimorphisms, but the large majority of the studies did not find differences in hemispheric asymmetries in males and females. 
A high discordance between results emerged in reviewing studies on structural asymmetries. The discordance is not explainable by a low number of participants because most studies had large sample sizes. Furthermore, the participant numbers between the studies may differ significantly. Brain asymmetries may be quite small, depending on the measurement (for example, see Kong et al. 2018), which in turn might lead to differences between studies, especially when the sample sizes differ. The temporal lobe is the most studied cerebral structure - the first neuropathological reports describe a larger left temporal hemisphere. Despite incomplete agreement between studies, evidence supports a larger planum temporale on the left side and a deeper superior temporal sulcus on the right. It has been reported that during primary gyrogenesis, the right superior temporal sulcus undergoes cortical folding earlier (Rajagopalan et al. 2011, 2012; Kasprian et al. 2011; Habas et al. 2012) and shows larger gyral complexity (Dubois et al. 2008, 2010). In addition, here, differences in sample size may perhaps justify some differences between studies.

The temporal planum on the left is often included in the Wernicke's area (Tremblay and Dick 2016), responsible for understanding spoken language. These data are in line with studies on the adult brain, showing that the temporal planum is more pronounced on the left than on the right in most individuals (Geschwind and Levitsky 1968).

Most results on the parieto-occipital cortex and subcortical grey matter exhibit a rightward asymmetry in full-term and premature infants. Brain structures with slower developmental rates, such as white matter and frontal lobes, have been poorly investigated, and the results are even more discordant.

Studies during gestation are scarce $(n=11)$, and all but one structural. Compared to studies on premature infants, those on foetuses are mainly conducted in earlier gestational weeks (late second and initial third trimester of gestation vs. the end of the third), when the brain is very immature. Therefore, anatomical investigations are less refined. As asymmetry emergence is mainly characterized by enlarging of the regions surrounding the Sylvian fissure in the left hemisphere (Dubois et al. 2008, 2010; Liu et al. 2010; Habas et al. 2012), these studies usually detected only a global enlargement of the left hemisphere. Results agree substantially with the first reports on the left-sided temporal lobe being significantly larger in post-mortem foetuses (Witelson and Pallie 1973; Chi et al. 1977), a morphological asymmetry already present from the 29th week of gestation (Wada et al. 1975).

Unlike data on structural asymmetries, functional data obtained in full-term infants, premature infants, and foetuses show a more harmonious pattern of results. Studies converge to identify a leftward dominance for speech stimuli; interestingly, this functional asymmetry correlates with communicative abilities at 6 and 12 months (Maitre et al. 2014), supporting the specificity of the left temporal lobe for language.

The other main finding of our revision is an overall dominance of the right hemisphere in all other functional conditions: sensory stimulations, non-linguistic characteristics of speech, and endogenous activity obtained during a resting state. The dominance of the right hemisphere for all conditions except linguistic stimuli is in line with the righthemisphere conservatism theory (Geschwind and Galaburda 1985), stating that the right hemisphere develops earlier and that its development is, therefore, less subject to external influences. The delay in maturation of the left hemisphere may allow higher plasticity in terms of environmental stimulation, such as language exposure and motor movements (Dubois et al. 2008). Compelling support has also been provided by Sun et al. (2005), who found significant asymmetries of gene expression in embryos as early as 12 -week gestational age.

In humans, as in animals, the right hemisphere sustains those functions necessary to survive, including visuospatial or emotional processes, which render its early development adaptive (Geschwind and Galaburda 1985). It has been shown, for example, that the right hemisphere systematically prevails over the left hemisphere in recognition of faces and facial expressions, mental rotation, and para-verbal stimuli, such as prosody and recognition of the connotative and affective tone of spoken language (see, e.g., George et al. 1996). Furthermore, right lateralization is established for the ventral frontoparietal network, which acts as a detector of relevant stimuli (especially if salient and unexpected) in a model by Corbetta and Shulman (2002). All these functions are crucial for a neonate.

Initial investigations of hemispheric asymmetry consisted of post-mortem explorations of aborted foetuses or dead newborns. Advances have come more quickly in the era of neuroimaging, overcoming the problem of small sample sizes and increasing the data availability also of healthy subjects; however, the increase in available data has rather complicated the evidence. Our data suggest that functional asymmetry regarding language is correlated with the perisylvian regions' structural asymmetry, but other associations between structural and functional findings are hard to establish. It is noteworthy that the relationship between structural and functional asymmetries is still far from being fully characterized also in adult studies (Dos Santos Sequeira et al. 2006). Rather more in agreeance are results on functionally critical morphological asymmetries, such as microstructural organization. For example, dendritic arborisation is usually greater in the language areas of left hemisphere than in the corresponding areas on the right (Scheibel et al. 1985).

Our review also highlights another dissociation between structural and functional results: while a broad agreement 
was found in functional studies, structural findings showed a low concordance among themselves. This appears particularly curious, considering the higher variability in techniques (EEG, fMRI, ERP, EP, NIRS, and MEG) and conditions (at rest or under stimulation) used in functional studies compared to structural ones. The reason is unclear, but functional studies may allow the cerebral functionality to emerge using appropriate tasks targeted to the immature brain. On the other side, structural investigations are static photographs of the whole brain. They provide information on the areas that mature later and exhibit higher plasticity in terms of experience, which may justify a higher inter-individual variability.

Interestingly, studies on older children and adults born prematurely have shown that volumetric and microstructural abnormalities are scarcely associated with neurodevelopment outcomes (Nosarti et al. 2008; Mathur et al. 2010; Seghier and Hüppi 2010). In contrast, functional connectivity data have been highly correlated with intelligence and task performance measures (Seeley et al. 2007; Van Den Heuvel et al. 2009; Nosarti et al. 2009; Myers et al. 2010). Connectivity analysis has been suggested to be particularly revealing when assessing hemispheric specialization (Stephan et al. 2007).

Finally, our review of the literature showed that premature infants had altered asymmetry measures compared to full-term infants, also in the absence of other risk factors. These data are in line with other studies on the developmental trajectories of premature infants (Suppiej et al. 2015, 2017; Cainelli et al. 2020a, 2021). Furthermore, lateralization abnormalities have been shown to persist over the long term: prematurely born adolescents exhibit fundamental alterations in the cerebral lateralization for language that significantly correlate with language scores (Wilke et al. 2014; Scheinost et al. 2015). Lateralization is implicated in language development, handedness, and higher-order reasoning and processing (Steinmetz et al. 1991; Turner et al. 2015). Therefore, the study of early abnormalities may help explain typical neurodevelopment and the origin of disorders, given the increased vulnerability to many extrinsic and intrinsic influences at this developmental phase (Andersen 2003). Abnormalities in structural and functional lateralization are suspected of contributing to various neuropathologies in humans, as several neurodevelopmental pathologies, such as schizophrenia (Oertel-Knöchel and Linden 2011; Ribolsi et al. 2014), obsessive-compulsive disorder (Rao et al. 2015), autism spectrum disorder (Gabard-Durnam et al. 2015), attention-deficit and hyperactivity disorder (Sigi Hale et al. 2014), and dyslexia (Brandler and Paracchini 2014) are associated with atypical patterns of functional and structural asymmetries.

Scoping reviews are useful for examining emerging evidence when it is still unclear what other, more specific questions can be posed and valuably addressed by a more precise systematic review (for guidance, see Tricco et al. 2018; Munn et al. 2018). As such, scoping reviews cannot uncover the international evidence, confirm current practice/address or any variation/identify new practices, identify and investigate conflicting results, produce statements to guide decision-making, as systematic reviews do (Munn et al. 2018). In particular, the provision of implications for practice is a key feature of systematic reviews that lacks in scoping reviews, given its absence of an assessment of methodological limitations or risk of bias of the evidence.

Investigating the emergence of early asymmetries, scoping review is the best choice, given the current literature in this research field. We hope that our work may be the starting point for future research and systematic reviews, which may, respectively, address unexplored areas or systematically reviews specific questions. For example, our work highlights several fields that request further investigation: sexual dysmorphisms, the development of structural and functional brain asymmetries in healthy foetuses, the presence of asymmetries in less explored brain regions, such as frontal and subcortical structures.

In conclusion, all but one study agreed on the existence of hemispheric asymmetry as early as the first appearance of cerebral structures. Functional asymmetry for language is correlated with the structural asymmetry of perisylvian regions. However, studies do not agree on the developmental direction, and the structural locations of several other asymmetries emerged, while most consistent results came from functional data. Globally, data related to a general dominance of the right hemisphere, accompanied by a selective leftward dominance for language, are in line with the assumption of an early-maturing right hemisphere and less genetic control over the left hemisphere, which would be influenced more by the in utero environment (Geschwind and Galaburda 1985; Geschwind et al. 2002). Understanding normative development is necessary to understand abnormalities in diseases and how they affect early-life experiences. We are still far away from a clear understanding of developmental trajectories and the significance of potential disorders later in neurodevelopment.

Supplementary Information The online version contains supplementary material available at https://doi.org/10.1007/s00429-021-02256-1.

Author contributions PB had the idea for the article, performed the literature search, and revised the work critically. EC performed the literature search and data analysis and drafted the work.

Funding Open access funding provided by Università degli Studi di Padova within the CRUI-CARE Agreement. The present work was carried out within the scope of the research program Dipartimenti di Eccellenza (art.1, commi 314-337 legge 232/2016), which was supported by a grant from MIUR to the Department of General Psychology, University of Padua. 
Data availability Not applicable.

Code availability Not applicable.

\section{Declarations}

Conflict of interest The authors have no relevant financial or non-financial interests to disclose.

Ethics approval Not applicable.

Consent to participate Not applicable.

Consent for publication Not applicable.

Open Access This article is licensed under a Creative Commons Attribution 4.0 International License, which permits use, sharing, adaptation, distribution and reproduction in any medium or format, as long as you give appropriate credit to the original author(s) and the source, provide a link to the Creative Commons licence, and indicate if changes were made. The images or other third party material in this article are included in the article's Creative Commons licence, unless indicated otherwise in a credit line to the material. If material is not included in the article's Creative Commons licence and your intended use is not permitted by statutory regulation or exceeds the permitted use, you will need to obtain permission directly from the copyright holder. To view a copy of this licence, visit http://creativecommons.org/licenses/by/4.0/.

\section{References}

Andersen SL (2003) Trajectories of brain development: Point of vulnerability or window of opportunity? In: Neuroscience and Biobehavioral Reviews. Elsevier Ltd, pp 3-18

Andescavage NN, Du Plessis A, McCarter R et al (2017) Complex trajectories of brain development in the healthy human fetus. Cereb Cortex 27:5274-5283. https://doi.org/10.1093/cercor/bhw306

Arimitsu T, Minagawa Y, Yagihashi T et al (2018) The cerebral hemodynamic response to phonetic changes of speech in preterm and term infants: the impact of postmenstrual age. NeuroImage Clin 19:599-606. https://doi.org/10.1016/j.nicl.2018.05.005

Barlow SM, Jegatheesan P, Weiss S et al (2014) Amplitude-integrated EEG and range-EEG modulation associated with pneumatic orocutaneous stimulation in preterm infants. J Perinatol 34:213219. https://doi.org/10.1038/jp.2013.150

Barttfeld P, Abboud S, Lagercrantz H et al (2018) A lateral-tomesial organization of human ventral visual cortex at birth. Brain Struct Funct 223:3107-3119. https://doi.org/10.1007/ s00429-018-1676-3

Bouchon C, Nazzi T, Gervain J (2015) Hemispheric asymmetries in repetition enhancement and suppression effects in the newborn brain. PLoS One 10:. https://doi.org/https://doi.org/10.1371/ journal.pone. 0140160

Brandler WM, Paracchini S (2014) The genetic relationship between handedness and neurodevelopmental disorders. Trends Mol Med 20:83-90

Brunoni AR, Moffa AH, Fregni F et al (2016) Transcranial direct current stimulation for acute major depressive episodes: Metaanalysis of individual patient data. Br J Psychiatry 208:522-531

Cainelli E, Arrigoni F, Vedovelli L (2020a) White matter injury and neurodevelopmental disabilities: a cross-disease (dis)connection.
Prog Neurobiol 193:101845. https://doi.org/10.1016/j.pneurobio. 2020.101845

Cainelli E, Di Bono MG, Bisiacchi PS, Suppiej A (2020b) Electroencephalographic functional connectivity in extreme prematurity: a pilot study based on graph theory. Pediatr Res 87:753-759. https://doi.org/10.1038/s41390-019-0621-3

Cainelli E, Vedovelli L, Wigley ILCM et al (2021) Neonatal spectral EEG is prognostic of cognitive abilities at school age in premature infants without overt brain damage. Eur J Pediatr 180(3):909-918. https://doi.org/10.1007/s00431-020-03818-x

Chance SA (2014) The cortical microstructural basis of lateralized cognition: a review. Front Psychol 5:. https://doi.org/https://doi. org/10.3389/fpsyg.2014.00820

Chi JG, Dooling EC, Gilles FH (1977) Left-right asymmetries of the temporal speech areas of the human fetus. Arch Neurol 34:346348. https://doi.org/10.1001/archneur.1977.00500180040008

Chiarello C, Vazquez D, Felton A, McDowell A (2016) Structural asymmetry of the human cerebral cortex: regional and between-subject variability of surface area, cortical thickness, and local gyrification. Neuropsychologia 93:365-379. https:// doi.org/10.1016/j.neuropsychologia.2016.01.012

Cioni G, Pellegrinetti G (1982) Lateralization of sensory and motor functions in human neonates. Percept Mot Skills 54:11511158. https://doi.org/10.2466/pms.1982.54.3c.1151

Corbetta M, Shulman GL (2002) Control of goal-directed and stimulus-driven attention in the brain. Nat Rev Neurosci 3:201-215. https://doi.org/10.1038/nrn755

Corsi-Cabrera M, Cubero-Rego L, Ricardo-Garcell J, Harmony T (2020) Week-by-week changes in sleep EEG in healthy fullterm newborns. Sleep 43:. https://doi.org/https://doi.org/10. 1093/sleep/zsz261

Crowell DH, Jones RH, Kapuniai LE, Nakagawa JK (1973) Unilateral cortical activity in newborn humans: An early index of cerebral dominance? Science (80- ) 180:205-207. https://doi. org/https://doi.org/10.1126/science.180.4082.205

Daneshvarfard F, Abrishami Moghaddam H, Dehaene-Lambertz G, et al (2019) Neurodevelopment and asymmetry of auditoryrelated responses to repetitive syllabic stimuli in preterm neonates based on frequency-domain analysis. Sci Rep 9:. https:// doi.org/https://doi.org/10.1038/s41598-019-47064-0

Dean DC, Planalp EM, Wooten W et al (2018) Investigation of brain structure in the 1-month infant. Brain Struct Funct 223:19531970. https://doi.org/10.1007/s00429-017-1600-2

Dos Santos Sequeira S, Woerner W, Walter C et al (2006) Handedness, dichotic-listening ear advantage, and gender effects on planum temporale asymmetry - a volumetric investigation using structural magnetic resonance imaging. Neuropsychologia 44:622-636. https://doi.org/10.1016/j.neuropsychologia. 2005.06.014

Duan D, Xia S, Rekik I et al (2019) Exploring folding patterns of infant cerebral cortex based on multi-view curvature features: Methods and applications. Neuroimage 185:575-592. https:// doi.org/10.1016/j.neuroimage.2018.08.041

Dubois J, Benders M, Cachia A et al (2008) Mapping the early cortical folding process in the preterm newborn brain. Cereb Cortex 18:1444-1454. https://doi.org/10.1093/cercor/bhm180

Dubois J, Benders M, Lazeyras F et al (2010) Structural asymmetries of perisylvian regions in the preterm newborn. Neuroimage 52:32-42. https://doi.org/10.1016/j.neuroimage.2010.03.054

Eiselt M, Schendel M, Witte H et al (1997) Quantitative analysis of discontinuous EEG in premature and full-term newborns during quiet sleep. Electroencephalogr Clin Neurophysiol 103:528-534. https://doi.org/10.1016/S0013-4694(97)00033-3

Esteves M, Moreira PS, Marques P, et al (2019) Asymmetrical subcortical plasticity entails cognitive progression in older 
individuals. Aging Cell 18:. https://doi.org/https://doi.org/10. 1111/acel.12857

Esteves M, Ganz E, Sousa N, Leite-Almeida H (2020) Asymmetrical Brain Plasticity: Physiology and Pathology. Neuroscience

Fox NA, Davidson RJ (1986) Taste-elicited changes in facial signs of emotion and the asymmetry of brain electrical activity in human newborns. Neuropsychologia 24:417-422. https://doi.org/10. 1016/0028-3932(86)90028-X

Fu L, Wang Y, Fang H et al (2020) Longitudinal study of brain asymmetries in autism and developmental delays aged 2-5 years. Neuroscience 432:137-149. https://doi.org/10.1016/j.neuroscience. 2020.02.028

Gabard-Durnam L, Tierney AL, Vogel-Farley V et al (2015) Alpha asymmetry in infants at risk for autism spectrum disorders. J Autism Dev Disord 45:473-480. https://doi.org/10.1007/ s10803-013-1926-4

George MS, Parekh PI, Rosinsky N et al (1996) Understanding emotional prosody activates right hemisphere regions. Arch Neurol 53:665-670. https://doi.org/10.1001/archneur.1996.0055007010 3017

Geschwind N, Galaburda AM (1985) Cerebral lateralization: biological mechanisms, associations, and pathology: III. A hypothesis and a program for research. Arch Neurol 42:634-654. https://doi.org/ 10.1001/archneur.1985.04060070024012

Geschwind N, Levitsky W (1968) Human brain: Left-right asymmetries in temporal speech region. Science (80- ) 161:186-187. https://doi.org/https://doi.org/10.1126/science.161.3837.186

Geschwind DH, Miller BL, DeCarli C, Carmelli D (2002) Heritability of lobar brain volumes in twins supports genetic models of cerebral laterality and handedness. Proc Natl Acad Sci USA 99:3176-3181. https://doi.org/10.1073/pnas.052494999

Gilmore JH, Zhai G, Wilber K et al (2004) 3 Tesla magnetic resonance imaging of the brain in newborns. Psychiatry Res-Neuroimaging 132:81-85. https://doi.org/10.1016/j.pscychresns.2004.04. 004

Gilmore JH, Lin W, Prastawa MW et al (2007) Regional gray matter growth, sexual dimorphism, and cerebral asymmetry in the neonatal brain. J Neurosci 27:1255-1260. https://doi.org/10.1523/ JNEUROSCI.3339-06.2007

González JJ, Mañas S, De Vera L et al (2011) Assessment of electroencephalographic functional connectivity in term and preterm neonates. Clin Neurophysiol 122:696-702. https://doi.org/10. 1016/j.clinph.2010.08.025

Gordon HW (2016) Laterality of brain activation for risk factors of addiction. Curr Drug Abuse Rev 9:1-18. https://doi.org/10.2174/ 1874473709666151217121309

Guo T, Winterburn JL, Pipitone J et al (2015) Automatic segmentation of the hippocampus for preterm neonates from early-in-life to term-equivalent age. NeuroImage Clin 9:176-193. https://doi. org/10.1016/j.nicl.2015.07.019

Habas PA, Scott JA, Roosta A et al (2012) Early folding patterns and asymmetries of the normal human brain detected from in utero MRI. Cereb Cortex 22:13-25. https://doi.org/10.1093/cercor/ bhr053

Habib R, Nyberg L, Tulving E (2003) Hemispheric asymmetries of memory: The HERA model revisited. Trends Cogn Sci 7:241-245

Hering-Hanit R, Achiron R, Lipitz S, Achiron A (2001) Asymmetry of fetal cerebral hemispheres: in utero ultrasound study. Arch Dis Child Fetal Neonatal Ed 85:f194-f196. https://doi.org/10. 1136/fn.85.3.f194

Hill J, Dierker D, Neil J et al (2010) A surface-based analysis of hemispheric asymmetries and folding of cerebral cortex in term-born human infants. J Neurosci 30:2268-2276. https://doi.org/10. 1523/JNEUROSCI.4682-09.2010
Hirnstein M, Hugdahl K, Hausmann M (2014) How brain asymmetry relates to performance - A large-scale dichotic listening study. Front Psychol 4:. https://doi.org/https://doi.org/10.3389/fpsyg. 2013.00997

Kaminska A, Delattre V, Laschet J et al (2018) Cortical auditoryevoked responses in preterm neonates: revisited by spectral and temporal analyses. Cereb Cortex 28:3429-3444. https://doi.org/ 10.1093/cercor/bhx206

Kasprian G, Langs G, Brugger PC et al (2011) The prenatal origin of hemispheric asymmetry: an in utero neuroimaging study. Cereb Cortex 21:1076-1083. https://doi.org/10.1093/cercor/bhq179

Kivilevitch Z, Achiron R, Zalel Y (2010) Fetal brain asymmetry: in utero sonographic study of normal fetuses. Am J Obstet Gynecol 202:359.e1-8. https://doi.org/10.1016/j.ajog.2009.11.001

Kong XZ, Mathias SR, Guadalupe T et al (2018) Mapping cortical brain asymmetry in 17,141 healthy individuals worldwide via the ENIGMA consortium. Proc Natl Acad Sci USA 115:E5154 E5163. https://doi.org/10.1073/pnas.1718418115

Kostović I, Judaš M (2006) Prolonged coexistence of transient and permanent circuitry elements in the developing cerebral cortex of fetuses and preterm infants. Dev Med Child Neurol 48:388-393

Kotilahti K, Nissilä I, Näsi T et al (2010) Hemodynamic responses to speech and music in newborn infants. Hum Brain Mapp 31:595603. https://doi.org/10.1002/hbm.20890

Kwon SH, Scheinost D, Lacadie C et al (2015) Adaptive mechanisms of developing brain: cerebral lateralization in the prematurelyborn. Neuroimage 108:144-150. https://doi.org/10.1016/j.neuro image.2014.12.032

Lehtola SJ, Tuulari JJ, Karlsson L et al (2019) Associations of age and sex with brain volumes and asymmetry in 2-5-week-old infants. Brain Struct Funct 224:501-513. https://doi.org/10.1007/ s00429-018-1787-x

LeMay M (1976) Morphological cerebral asymmetries of modern man, fossil man, and nonhuman primate. Ann NY Acad Sci 280:349-366

Li G, Nie J, Wang L et al (2014) Mapping longitudinal hemispheric structural asymmetries of the human cerebral cortex from birth to 2 years of age. Cereb Cortex 24:1289-1300. https://doi.org/ $10.1093 /$ cercor/bhs413

Li G, Liu T, Ni D et al (2015) Spatiotemporal patterns of cortical fiber density in developing infants, and their relationship with cortical thickness. Hum Brain Mapp 36:5183-5195. https://doi.org/ $10.1002 / \mathrm{hbm} .23003$

Lin P-Y, Dehaes M, Roche-Labarbe N et al (2012) Regional and hemispheric asymmetries of cerebral hemodynamic and oxygen metabolism in newborns. Biomed Opt Biomed 23:339-348. https://doi.org/10.1364/biomed.2012.bsu4a.6

Lin P-Y, Roche-Labarbe N, Dehaes M et al (2013) Regional and hemispheric asymmetries of cerebral hemodynamic and oxygen metabolism in newborns. Cereb Cortex 23:339-348. https://doi. org/10.1093/cercor/bhs023

Liu Y, Balériaux D, Kavec M et al (2010) Structural asymmetries in motor and language networks in a population of healthy preterm neonates at term equivalent age: a diffusion tensor imaging and probabilistic tractography study. Neuroimage 51:783-788. https://doi.org/10.1016/j.neuroimage.2010.02.066

Maitre NL, Slaughter JC, Aschner JL, Key AP (2014) Hemisphere differences in speech-sound event-related potentials in intensive care neonates: associations and predictive value for development in infancy. J Child Neurol 29:903-911. https://doi.org/10.1177/ 0883073813493502

Mathur AM, Neil JJ, Inder TE (2010) Understanding brain injury and neurodevelopmental disabilities in the preterm infant: the evolving role of advanced magnetic resonance imaging. Semin Perinatol 34:57-66 
Matsuzawa J, Matsui M, Konishi T et al (2001) Age-related volumetric changes of brain gray and white matter in healthy infants and children. Cereb Cortex 11:335-342. https://doi.org/10.1093/ cercor/11.4.335

McCartney G, Hepper P (1999) Development of lateralized behaviour in the human fetus from 18 to 27 weeks' gestation. Dev Med Child Neurol 41:83-86. https://doi.org/10.1017/S001216229 9000183

Mento G, Suppiej A, Altoè G, Bisiacchi PS (2010) Functional hemispheric asymmetries in humans: electrophysiological evidence from preterm infants. Eur J Neurosci 31:565-574. https://doi.org/ 10.1111/j.1460-9568.2010.07076.x

Minagawa-Kawai Y, Cristià A, Vendelin I, et al (2011) Assessing signal-driven mechanisms in neonates: Brain responses to temporally and spectrally different sounds. Front Psychol 2:. https:// doi.org/https://doi.org/10.3389/fpsyg.2011.00135

Molfese DL, Nunez V, Seibert SM, Ramanaiah NV (1976) Cerebral asymmetry: changes in factors affecting its development. Ann N Y Acad Sci 280:821-833

Munn Z, Peters MDJ, Stern C, et al (2018) Systematic review or scoping review? Guidance for authors when choosing between a systematic or scoping review approach. BMC Med Res Methodol 18:. https://doi.org/https://doi.org/10.1186/s12874-018-0611-x

Myers EH, Hampson M, Vohr B et al (2010) Functional connectivity to a right hemisphere language center in prematurely born adolescents. Neuroimage 51:1445-1452. https://doi.org/10.1016/j. neuroimage.2010.03.049

Myers MM, Grieve PG, Izraelit A et al (2012) Developmental profiles of infant EEG: overlap with transient cortical circuits. Clin Neurophysiol 123:1502-1511. https://doi.org/10.1016/j.clinph. 2011.11.264

Nosarti C, Giouroukou E, Healy E et al (2008) Grey and white matter distribution in very preterm adolescents mediates neurodevelopmental outcome. Brain 131:205-217. https://doi.org/10.1093/ brain/awm 282

Nosarti C, Shergill SS, Allin MP et al (2009) Neural substrates of letter fluency processing in young adults who were born very preterm: Alterations in frontal and striatal regions. Neuroimage 47:19041913. https://doi.org/10.1016/j.neuroimage.2009.04.041

Ocklenburg S, Friedrich P, Güntürkün O, Genç E (2016) Intrahemispheric white matter asymmetries: The missing link between brain structure and functional lateralization? Rev Neurosci 27:465-480

Oertel-Knöchel V, Linden DEJ (2011) Cerebral asymmetry in schizophrenia. Neuroscientist 17:456-467. https://doi.org/10.1177/ 1073858410386493

Peña M, Maki A, Kovačić D et al (2003) Sounds and silence: an optical topography study of language recognition at birth. Proc Natl Acad Sci USA 100:11702-11705. https://doi.org/10.1073/pnas. 1934290100

Perani D, Saccuman MC, Scifo P et al (2011) Neural language networks at birth. Proc Natl Acad Sci USA 108:16056-16061. https://doi.org/10.1073/pnas.1102991108

Rajagopalan V, Scott J, Habas PA et al (2011) Local tissue growth patterns underlying normal fetal human brain gyrification quantified in utero. J Neurosci 31:2878-2887. https://doi.org/10.1523/ JNEUROSCI.5458-10.2011

Rajagopalan V, Scott J, Habas PA et al (2012) Mapping directionality specific volume changes using tensor based morphometry: An application to the study of gyrogenesis and lateralization of the human fetal brain. Neuroimage 63:947-958. https://doi.org/10. 1016/j.neuroimage.2012.03.092

Rao NP, Arasappa R, Reddy NN et al (2015) Lateralisation abnormalities in obsessive-compulsive disorder: a line bisection study. Acta Neuropsychiatr 27:242-247. https://doi.org/10.1017/neu.2015.23
Ratnarajah N, Rifkin-Graboi A, Fortier MV et al (2013) Structural connectivity asymmetry in the neonatal brain. Neuroimage 75:187-194. https://doi.org/10.1016/j.neuroimage.2013.02.052

Ribolsi M, Daskalakis ZJ, Siracusano A, Koch G (2014) Abnormal asymmetry of brain connectivity in schizophrenia. Front Hum Neurosci 8:. https://doi.org/https://doi.org/10.3389/fnhum.2014. 01010

Scheibel AB, Paul LA, Fried I et al (1985) Dendritic organization of the anterior speech area. Exp Neurol 87:109-117. https://doi.org/ 10.1016/0014-4886(85)90137-2

Scheinost D, Lacadie C, Vohr BR et al (2015) Cerebral lateralization is protective in the very prematurely born. Cereb Cortex 25:18581866. https://doi.org/10.1093/cercor/bht430

Scher MS, Steppe DA, Sclabassi RJ, Banks DL (1997) Regional differences in spectral EEG measures between healthy term and preterm infants. Pediatr Neurol 17:218-223. https://doi.org/10. 1016/S0887-8994(97)00101-X

Schleussner E, Schneider U, Arnscheidt C et al (2004) Prenatal evidence of left-right asymmetries in auditory evoked responses using fetal magnetoencephalography. Early Hum Dev 78:133136. https://doi.org/10.1016/j.earlhumdev.2004.03.005

Schmitz J, Fraenz C, Schlüter C et al (2019) Hemispheric asymmetries in cortical gray matter microstructure identified by neurite orientation dispersion and density imaging. Neuroimage 189:667-675. https://doi.org/10.1016/j.neuroimage.2019. 01.079

Scott JA, Habas PA, Kim K et al (2011) Growth trajectories of the human fetal brain tissues estimated from 3D reconstructed in utero MRI. Int J Dev Neurosci 29:529-536. https://doi.org/10. 1016/j.ijdevneu.2011.04.001

Seeley WW, Menon V, Schatzberg AF et al (2007) Dissociable intrinsic connectivity networks for salience processing and executive control. J Neurosci 27:2349-2356. https://doi.org/10.1523/JNEUR OSCI.5587-06.2007

Seghier ML, Hüppi PS (2010) The role of functional magnetic resonance imaging in the study of brain development, injury, and recovery in the newborn. Semin Perinatol 34:79-86

Seidenwurm D, Roger Bird C, Enzmann DR, Marshall WH (1985) Left-right temporal region asymmetry in infants and children. Am J Neuroradiol 6:777-779

Shaw P, Lalonde F, Lepage C et al (2009) Development of cortical asymmetry in typically developing children and its disruption in attention-deficit/hyperactivity disorder. Arch Gen Psychiatry 66:888-896. https://doi.org/10.1001/archgenpsychiatry.2009.103

Sigi Hale T, Kane AM, Kaminsky O, et al (2014) Visual network asymmetry and default mode network function in ADHD: An fMRI study. Front Psychiatry 5:. https://doi.org/https://doi.org/10.3389/ fpsyt.2014.00081

Song D, Jegatheesan P, Weiss S et al (2014) Modulation of EEG spectral edge frequency during patterned pneumatic oral stimulation in preterm infants. Pediatr Res 75:85-92. https://doi.org/10.1038/ pr.2013.179

Song JW, Mitchell PD, Kolasinski J et al (2015) Asymmetry of white matter pathways in developing human brains. Cereb Cortex 25:2883-2893. https://doi.org/10.1093/cercor/bhu084

Sowell ER, Thompson PM, Rex D et al (2002) Mapping sulcal pattern asymmetry and local cortical surface gray matter distribution in vivo: Maturation in perisylvian cortices. Cereb Cortex 12:17-26. https://doi.org/10.1093/cercor/12.1.17

Steinmetz H, Volkmann J, Jäncke L, Freund H-J (1991) Anatomical left-right asymmetry of language-related temporal cortex is different in left- and right-handers. Ann Neurol 29:315-319. https:// doi.org/10.1002/ana.410290314

Stephan KE, Fink GR, Marshall JC (2007) Mechanisms of hemispheric specialization: insights from analyses of connectivity. Neuropsychologia 45:209-228 
Sun T, Patoine C, Abu-Khalil A, et al (2005) Neuroscience: Early asymmetry of gene transcription in embryonic human left and right cerebral cortex. Science (80- ) 308:1794-1798. https://doi. org/https://doi.org/10.1126/science.1110324

Suppiej A, Cappellari A, Cainelli E (2012) Clinical neurophysiology in preterm infants: A window on early phases of brain development. In: Pediatric Neurology. Nova Publisher, pp 115-130

Suppiej A, Cainelli E, Cappellari A et al (2015) Neonatal cortical auditory evoked potentials are affected by clinical conditions occurring in early prematurity. J Clin Neurophysiol 32:419-423. https://doi.org/10.1097/WNP.0000000000000182

Suppiej A, Cainelli E, Cappellari A et al (2017) Spectral analysis highlight developmental EEG changes in preterm infants without overt brain damage. Neurosci Lett 649:112-115. https://doi.org/ 10.1016/j.neulet.2017.04.021

Tan U, Zor N, Kücüközkan T et al (1993) Grasp reflex strength from right and left hands is associated with ph stressor from the umbilical arterial blood in human newborns: handedness and sex-related differences. Int J Neurosci 72:149-156. https://doi. org/10.3109/00207459309024103

Telkemeyer S, Rossi S, Koch SP et al (2009) Sensitivity of newborn auditory cortex to the temporal structure of sounds. J Neurosci 29:14726-14733. https://doi.org/10.1523/JNEUROSCI.1246-09. 2009

Telpaz A, Yechiam E (2014) Contrasting losses and gains increases the predictability of behavior by frontal EEG asymmetry. Front Behav Neurosci 8:. https://doi.org/https://doi.org/10.3389/fnbeh. 2014.00149

Thiebaut de Schotten M, ffytche DH, Bizzi A, et al (2011) Atlasing location, asymmetry and inter-subject variability of white matter tracts in the human brain with MR diffusion tractography. Neuroimage 54:49-59. https://doi.org/10.1016/j.neuroimage. 2010.07.055

Thompson DK, Wood SJ, Doyle LW et al (2009) MR-determined hippocampal asymmetry in full-term and preterm neonates. Hippocampus 19:118-123. https://doi.org/10.1002/hipo.20492

Tremblay P, Dick AS (2016) Broca and wernicke are dead, or moving past the classic model of language neurobiology. Brain Lang 162:60-71

Tricco AC, Lillie E, Zarin W et al (2018) PRISMA extension for scoping reviews (PRISMA-ScR): checklist and explanation. Ann Intern Med 169:467-473

Turner BO, Marinsek N, Ryhal E, Miller MB (2015) Hemispheric lateralization in reasoning. Ann NY Acad Sci 1359:47-64. https:// doi.org/10.1111/nyas. 12940

Van Den Heuvel MP, Stam CJ, Kahn RS, Hulshoff Pol HE (2009) Efficiency of functional brain networks and intellectual performance.
J Neurosci 29:7619-7624. https://doi.org/10.1523/JNEUROSCI. 1443-09.2009

Vannucci RC, Heier LA, Vannucci SJ (2019) Cerebral asymmetry during development using linear measures from MRI. Early Hum Dev 139:. https://doi.org/https://doi.org/10.1016/j.earlhumdev. 2019.104853

Vasung L, Rollins CK, Yun HJ et al (2020) Quantitative in vivo MRI Assessment of Structural Asymmetries and Sexual Dimorphism of Transient Fetal Compartments in the Human Brain. Cereb Cortex 30:1752-1767. https://doi.org/10.1093/cercor/bhz200

Wada JA (1977) Pre-language and fundamental asymmetry of the infant brain Evolution and lateralization of the brain. Ann NY Acad Sci 299:370-378

Wada JA, Clarke R, Hamm A (1975) Cerebral hemispheric asymmetry in humans: cortical speech zones in 100 adult and 100 infant brains. Arch Neurol 32:239-246. https://doi.org/10.1001/archn eur.1975.00490460055007

Wilke M, Hauser TK, Krägeloh-Mann I, Lidzba K (2014) Specific impairment of functional connectivity between language regions in former early preterms. Hum Brain Mapp 35:3372-3384. https://doi.org/10.1002/hbm.22408

Wilkinson M, Kane T, Wang R, Takahashi E (2017a) Migration pathways of thalamic neurons and development of thalamocortical connections in humans revealed by diffusion MR tractography. Cereb Cortex 27:5683-5695. https://doi.org/10.1093/cercor/ bhw339

Wilkinson M, Lim AR, Cohen AH, et al (2017b) Detection and growth pattern of arcuate fasciculus from newborn to adult. Front Neurosci 11:. https://doi.org/https://doi.org/10.3389/fnins.2017.00389

Witelson SF, Pallie W (1973) Left hemisphere specialization for language in the newborn: neuroanatomical evidence of asymmetry. Brain 96:641-646. https://doi.org/10.1093/brain/96.3.641

Zago L, Petit L, Jobard G et al (2017) Pseudoneglect in line bisection judgement is associated with a modulation of right hemispheric spatial attention dominance in right-handers. Neuropsychologia 94:75-83. https://doi.org/10.1016/j.neuropsychologia.2016.11. 024

Zhen Z, Yang Z, Huang L et al (2015) Quantifying interindividual variability and asymmetry of face-selective regions: a probabilistic functional atlas. Neuroimage 113:13-25. https://doi.org/10. 1016/j.neuroimage.2015.03.010

Publisher's Note Springer Nature remains neutral with regard to jurisdictional claims in published maps and institutional affiliations. 